\title{
Clinical Significance of Vertebral Artery Hypoplasia in Lateral Medullary Infarction
}

\section{Hyung Geun Oh, Seung Chul Lee}

Stroke and Cerebrovascular Center, Department of Neurology, Soonchunhyang University Cheonan Hospital, Soonchunhyang University College of Medicine, Cheonan, Korea

\begin{abstract}
Objective: The clinical significance of vertebral artery hypoplasia (VAH) and the possible pathomechanism of lateral medullary infarction in patients with VAH are still not completely clear.

Methods: Among 3,968 patients with acute ischemic stroke, we selected 102 patients with lateral medullary infarction (2.6\% [102/ 3,968]; 67 men, 35 women; mean age, 66 years; range, 33 to 86 years) who underwent brain magnetic resonance imaging, contrastenhanced magnetic resonance angiography, and transcranial Doppler (TCD) within 1 week of symptom onset. We compared the characteristics of VAH and non-VAH group.

Results: Of 102 patients with lateral medullary infarction, 34 (33.3\%) had hypoplastic vertebral artery and 68 (66.7\%) were non-VAH. The location of stroke in patients with right-sided VAH $(\mathrm{n}=22)$ was predominantly ipsilateral and in the 12 patients with left-sided VAH, the stroke was predominantly ipsilateral. TCD parameters of Mean flow velocity and pulsatility index were significantly different on the affected side between VAH and non-VAH groups $(P<0.001)$. Patients having dominant vertebral artery is opposite to basilar artery curvature are $88.2 \%$ (30/34).

Conclusion: We concluded that VAH can be considered an additional risk factor for lateral medullary infarction. The influence of reduced blood flow in the hypoplastic vertebral artery is also important to consider, especially when other vascular risk factor are present. The presence of dolichoectasy of the basilar artery ipsilateral to the VAH causes reorganization of the blood flow around the vertebrobasilar junction.
\end{abstract}

Keywords: Vertebral artery; Hypoplasia; Infarction

\section{INTRODUCTION}

Vertebral artery hypoplasia (VAH) was described in the 19th century. Congenital variations of the caliber of the vertebral arteries or asymmetry of these vessels are not infrequent. VAH with caliber discrepancies of more than 1:1.7 was observed in up to $10 \%$ of normal individuals. There is a tendency for the contralateral vessel to compensate with an increased diameter of about $5 \mathrm{~mm}$. There is no general agreement as to the definition of VAH. Operational definitions of VAH vary between diameters of less than 2 to less than $3 \mathrm{~mm}$ or an asymmetry ratio of equal or greater than 1:1.7. Additionally, reduced diastolic flow velocity associated with increased resistance index values indicates VAH $[1,2]$.

Acquired morphological variations in the vertebral artery (VA), such as stenosis, occlusion, or dissection, are associated with different clinical symptoms. The clinical significance of VAH is not currently well understood. In the literature, a possible causal association between the presence of VAH and the incidence of posterior stroke has been shown. Sonographic finding of VAH has reported the presence of reduced flow velocities (compared to the contralateral side), and high resistance flow pattern in the Doppler spectrum. However, the exact pathomechanism of ischemic stroke in patients with VAH is still not completely clear [3-7].

Lateral medullary infarction (Wallenberg syndrome) is the clinical manifestation resulting from occlusion of the intracranial vertebral artery (ICVA) or of the posterior inferior cerebellar artery (PICA) or one of its branches, in which the lateral part of the medulla oblongata infarcts, resulting in a typical pattern. The most 
commonly affected artery is the ICVA, followed by the PICA [8].

The aim of our study was to investigate clinical significance of $\mathrm{VAH}$ and the possible pathomechanism of lateral medullary infarction in patients with VAH.

\section{MATERIALS AND METHODS}

Participants were selected from 3,968 consecutive patients with acute ischemic stroke who were admitted to Soonchunhyang University Cheonan Hospital between September 2007 and August 2017. Among 3,968 patients, we selected 102 patients with lateral medullary infarction (2.6\% [102/3,968]; 67 men, 35 women; mean age, 66 years; range, 33 to 86 years) who underwent brain magnetic resonance imaging, contrast-enhanced magnetic resonance angiography (MRA), and transcranial Doppler (TCD) within 1 week of symptom onset. The acute ischemic lesion on lateral medulla was assessed by high signal intensity on diffusion-weighted imaging with low signal intensity on apparent diffusion coefficient. MRA was analyzed blindly to the TCD results. VA was classified as hypoplasia and non-hypoplasia according to our MRA criteria.

VAH was defined to have a lumen diameter of less than 2-3 mm. Because there is no consensus on the value of VAH, we defined $\mathrm{VAH}$ as a diameter of $\leq 2.2 \mathrm{~mm}$ or an asymmetry ratio of equal or greater than 1:1.7. For defining the diameter of VAH, we examined the V2 of the largest VA by using contrast-enhanced MRA. The location of the VA was defined according to standard criteria (V1, prevertebral portion; V2, the portion within the vertebral columns; V3, atlantoaxial portion; V4, intracranial portion) [8]. To assess reliability, the MRA of each patient was independently reviewed by two investigators ( $\mathrm{HG}$ Oh, SC Lee), both of whom were blinded to all clinical information. The consensus for the presence of VAH was standardized according to neuro-radiological interpretation reports and was acceptable $(\kappa=0.85, \mathrm{P}<0.01)$. In nonVAH group, stenosis degree of the distal VA (V4) responsible for lateral medullary infarction was defined as occlusion, stenosis ( $>50 \%$ reduction of arterial column width) and normal. Also we investigated the direction and degree of basilar artery (BA) curvature and relationship between VA dominance and BA dolichosis.

We assessed the risk factors in each patient for acute ischemic stroke, such as hypertension, diabetes, hyperlipidemia, current smoking, and heart disease. Cardiogenic embolic diseases (e.g., atrial fibrillation, left ventricular akinesia, recent myocardial infarction, or patent foramen ovale with right-to-left shunt) were routinely evaluated in all patients by electrocardiography and transthoracic echocardiography; further studies - such as transesophageal echocardiography or 24-hour holter monitoring - were performed if any abnormality was found on initial work-up. For each patient we attempted to determine the etiology of stroke.

TCD was performed with a $2-\mathrm{MHz}$ pulsed Doppler instrument by an experienced sonographer. VA was evaluated between 40 and $80 \mathrm{~mm}$ depth through suboccipital window. Mean flow velocity (MFV), pulsatility index (PI), and flow velocity ratio to BA (VA/BA FVR) were compared between VAH and non-VAH group. Asymmetry index (AI) of VA calculated by $2 \times 100 \times$ (ipsilateral MFVcontralateral MFV)/(ipsilateral MFV+contralateral MFV) (\%) was also analyzed.

Statistical analysis was done using SPSS ver. 17.0 (SPSS Inc., Chicago, IL, USA). Comparisons were performed using the Student ttest for continuous variables and Fisher's exact test for nominallevel variables. $\mathrm{P}<0.05$ was considered as significant.

\section{RESULTS}

Of 102 patients with lateral medullary infarction, 34 (33.3\%) had hypoplastic VA and 68 (66.7\%) were non-VAH. We found rightsided VAH more frequently, that is, in 22 cases (64.7\%). In 12 cases (35.3\%), the VAH was located on the left side. None of non-VAH patients had experienced a VA dissection.

There was no substantial age difference between the non-VAH and the VAH groups. The difference in gender between VAH and non-VAH group was also not statistically significant. There was no correlation between the conventional vascular risk factors (hypertension, diabetes mellitus, hyperlipidemia, and smoking) and the presence or absence of VAH in patients with lateral medullary infarction (Table 1).

The location of stroke in patients with right-sided VAH $(n=22)$ was predominantly ipsilateral and in the 12 patients with left-sided VAH, the stroke was predominantly ipsilateral (Table 2). Patients having dominant VA is opposite to BA curvature are $88.2 \%(30 / 34)$. These findings were assigned to the blood flow reorganization mechanism based on the findings of Hong et al. [9] (Fig. 1).

TCD parameters of MFV and PI were significantly different on the affected side between VAH and non-VAH groups. On the nonaffected side, MFV was significantly higher on VAH group. Also, AI was higher in VAH group, which was statistically significant (Table 3). 
Oh HG, et al. • Vertebral Artery Hypoplasia in Lateral Medullary Infarction

Table 1. Basic demographic characteristics and stroke risk factors of patients with lateral medullary infarction between $\mathrm{VAH}$ group and non-VAH group $(n=102)$

\begin{tabular}{lccc}
\hline Characteristic & $\begin{array}{c}\text { VAH group } \\
(\mathrm{n}=34)\end{array}$ & $\begin{array}{c}\text { Non-VAH group } \\
(\mathrm{n}=68)\end{array}$ & P-value $^{\mathrm{a})}$ \\
\hline Age (yr) & $65.5 \pm 10.3$ & $67.2 \pm 9.4$ & $\mathrm{NS}$ \\
Male sex & $24(70.6)$ & $43(63.2)$ & $\mathrm{NS}$ \\
Hypertension & $12(35.3)$ & $23(33.8)$ & $\mathrm{NS}$ \\
Diabetic mellitus & $3(8.8)$ & $7(10.3)$ & $\mathrm{NS}$ \\
Hyperlipidemia & $6(17.6)$ & $11(16.2)$ & $\mathrm{NS}$ \\
Smoking & $4(11.8)$ & $8(11.8)$ & $\mathrm{NS}$ \\
Cardioembolism & $4(11.8)$ & $10(14.7)$ & $\mathrm{NS}$ \\
\hline
\end{tabular}

Values are presented as mean \pm standard deviation or number (\%)

VAH, vertebral artery hypoplasia; NS, non-significant.

${ }^{a}$ Calculated by Student t-test for continuous variable and Fisher's exact test for categorical variable as appropriate.

Table 2. Laterality of VAH correlated to the location of ischemic lesion

\begin{tabular}{lcc}
\hline Location of stroke & Right sided VAH $(\mathrm{n}=22)$ & Left sided VAH $(\mathrm{n}=12)$ \\
\hline Right lateral medulla & 20 & 1 \\
Left lateral medulla & 2 & 11 \\
\hline
\end{tabular}

VAH, vertebral artery hypoplasia.

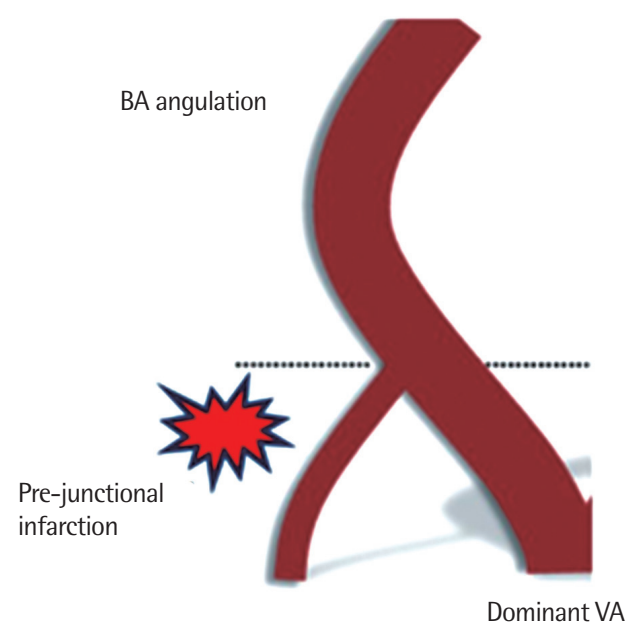

Fig. 1. Schematic illustration of the pathophysiological process of peri-vertebral junctional infarcts: possible changes in vertebrobasilar vessels under the condition of an unequal VA flow. Curving or elongation of BA caused by asymmetric wall tension. Non-dominant VA can lead to be narrow due to direct decrease of flow or easy collapse by Bernoulli's effect under the condition of decreased vascular remodeling capacity. Eventually, pre-junctional infarcts in non-dominant VA side. From Hong et al. J Neurol Neurosurg Psychiatry 2009;80:1087-92 [9]. $\mathrm{BA}$, basilar artery; VA, vertebral artery.

\section{DISCUSSION}

Recently, a cut-off point of $<2.2 \mathrm{~mm}$ was determined for $\mathrm{VAH}$, based on significant hemodynamic changes, which is further sup-
Table 3. MFV, PI, FVR to BA, and Al of vertebral artery

\begin{tabular}{lccc}
\hline Variable & $\begin{array}{c}\text { VAH group } \\
(\mathrm{n}=34)\end{array}$ & $\begin{array}{c}\text { Non-VAH group } \\
(\mathrm{n}=68)\end{array}$ & P-value $^{\text {a) }}$ \\
\hline Affected site & & & \\
MFV (cm/sec) & $26.5 \pm 13.1$ & $33.0 \pm 11.5$ & $<0.001$ \\
Pl & $0.91 \pm 0.20$ & $0.73 \pm 0.15$ & $<0.001$ \\
VA/BA FVR & $0.78 \pm 0.44$ & $0.77 \pm 0.28$ & $\mathrm{NS}$ \\
Non-affected site & & & \\
MFV (cm/sec) & $38.5 \pm 17.7$ & $29.5 \pm 12.5$ & $<0.001$ \\
Pl & $0.75 \pm 0.18$ & $0.74 \pm 0.15$ & $\mathrm{NS}$ \\
VA/BAFVR & $0.79 \pm 0.48$ & $0.78 \pm 0.31$ & $\mathrm{NS}$ \\
Al of the VA $(\%)$ & $24.5 \pm 22.3$ & $17.2 \pm 13.7$ & $<0.001$ \\
\hline
\end{tabular}

Values are presented as mean \pm standard deviation. $\mathrm{P}<0.05$ is considered statistically significant.

MFV, mean flow velocity; PI, pulsatility index; FVR, flow velocity ratio; $\mathrm{BA}$, basilar artery; Al, asymmetry index; VAH, vertebral artery hypoplasia; VA, vertebral artery; NS, non-significant.

a) Student t-test was used.

ported by an increase in ipsilateral flow resistance (resistance index $\geq 0.75$ ), contralateral diameter (side-to-side diameter difference $\geq 0.12 \mathrm{~mm}$ ), and flow volume (side-to-side flow volume ratio $\geq 5$ ) by color-coded duplex ultrasonography. The frequency of VAH (defined as VA diameter $<2.2 \mathrm{~mm}$ by duplex ultrasonography) was shown to be $11.6 \%$ (7.8\% on the right and $3.8 \%$ on the left) in 447 subjects [1,2]. In Korean study, the frequencies of VAH were as high as $35.2 \%$ in 529 patients with ischemic stroke (3-dimensional time-of-flight or contrast enhanced MRA) [4]. VAH is predominant on the right side, which is consistent with our finding.

VA hypoplasia as a congenital vessel variation was first mentioned in the 19th century. About a quarter of all ischemic strokes are localized in the vertebrobasilar circulation territory. Emerging evidences suggest that VA hypoplasia may contribute to posterior circulation ischemic events, especially when other risk factors coexist $[4,5,10]$.

In patients with lateral medullary infarction, the most common vascular lesion is occlusion of the proximal or middle portion of the ICVA. Penetrating branches to the lateral medulla arise from the middle and distal $2 / 3$ of the ICVAs and penetrate through the lateral medullary fossa to reach and supply the lateral medullary tegmentum. The medial branches of PICA supply only a small portion of the dorsal medullary tegmentum. The ICVA occlusive lesions decrease flow in these penetrators. Less often lateral medullary infarction is caused by occlusion of one of the small medullary branches [8].

It is assumed that the vessel diameter plays an important role in 
the ischemia mechanism. According to Poiseuille's law, the flow rate is proportional to the fourth power of the vessel radius. Based on the hemodynamics in VAH, the reduced blood flow in a hypoplastic VA plays an important role in the mechanism of stroke in the vertebrobasilar territory. The VAH in coexistence with known risk factors for stroke may increase their negative clinical impact. Hong et al. [9] postulated that the unequally mechanical forces resulting from the asymmetric VA flow might influence the morphological deformation in the vertebrobasilar arterial system, for example, a lateral displacement or elongation (dolichoectasy) of the BA The presence of dolichoectasy of the BA ipsilateral to the VAH causes reorganization of the blood flow around the vertebrobasilar junction. This BA deviation predisposes to increased frequency of stroke in the territory of the hypoplastic VA (Fig. 1) [9, $11,12]$.

Lateral medullary infarctions should be more frequent on the side of VAH. It is thought that luminal narrowing of the VAH might make it less feasible for cardiogenic emboli to pass through it. The $\mathrm{VAH}$, which shows lower mean flow volume and decreased flow velocities, seems to be more susceptible to pro-thrombotic or atherosclerotic processes than normal or dominant vertebral arteries. Vertebral arterial thrombi can cause in situ strokes on lateral medulla and prone to cause distal embolization to PICA territory lateral medulla $[12,13]$.

We concluded that VAH can be considered an additional risk factor for lateral medullary infarction when coexisting with conventional vascular risk factors. The influence of reduced blood flow in the hypoplastic VA is also important to consider, especially when other vascular risk factor are present.

Because of the relatively small sample size, further studies are necessary to confirm the association between VAH and lateral medullary infarction and to clarify the pathomechanism of stroke in the presence of VAH.

\section{REFERENCES}

1. Chuang YM, Chan L, Wu HM, Lee SP, Chu YT. The clinical relevance of vertebral artery hypoplasia. Acta Neurol Taiwan 2012;21:1-7.

2. Trattnig S, Schwaighofer B, Hubsch P, Schwarz M, Kainberger F. Colorcoded Doppler sonography of vertebral arteries. J Ultrasound Med 1991; 10:221-6.

3. Gaigalaite V, Vilimas A, Ozeraitiene V, Dementaviciene J, Janilionis R, Kalibatiene D, et al. Association between vertebral artery hypoplasia and posterior circulation stroke. BMC Neurol 2016;16:118.

4. Park JH, Kim JM, Roh JK. Hypoplastic vertebral artery: frequency and associations with ischaemic stroke territory. J Neurol Neurosurg Psychiatry 2007;78:954-8.

5. Perren F, Poglia D, Landis T, Sztajzel R. Vertebral artery hypoplasia: a predisposing factor for posterior circulation stroke? Neurology 2007;68:65-7.

6. Giannopoulos S, Markoula S, Kosmidou M, Pelidou SH, Kyritsis AP. Lateral medullary ischaemic events in young adults with hypoplastic vertebral artery. J Neurol Neurosurg Psychiatry 2007;78:987-9.

7. Chaturvedi S, Lukovits TG, Chen W, Gorelick PB. Ischemia in the territory of a hypoplastic vertebrobasilar system. Neurology 1999;52:980-3.

8. Caplan LR. The intracranial vertebral artery: a neglected species: the Johann Jacob Wepfer Award 2012. Cerebrovasc Dis 2012;34:20-30.

9. Hong JM, Chung CS, Bang OY, Yong SW, Joo IS, Huh K. Vertebral artery dominance contributes to basilar artery curvature and peri-vertebrobasilar junctional infarcts. J Neurol Neurosurg Psychiatry 2009;80:1087-92.

10. Katsanos AH, Kosmidou M, Kyritsis AP, Giannopoulos S. Is vertebral artery hypoplasia a predisposing factor for posterior circulation cerebral ischemic events?: a comprehensive review. Eur Neurol 2013;70:78-83.

11. Hademenos GJ, Massoud TF. Biophysical mechanisms of stroke. Stroke 1997;28:2067-77.

12. Szarazova AS, Bartels E, Bartels S, Turcani P. Possible morphological pathomechanisms of ischemic stroke in the posterior circulation of patients with vertebral artery hypoplasia. J Neuroimaging 2015;25:408-14.

13. Min JH, Lee YS. Transcranial Doppler ultrasonographic evaluation of vertebral artery hypoplasia and aplasia. J Neurol Sci 2007;260:183-7. 